\title{
Analysis of Pediatric Tendon Injuries in the Hand in Comparison with Adults
}

\author{
Jin Sung Kim ${ }^{1}$, Seung Je Sung ${ }^{2}$, Young Joon Kim², Young Woong Choi ${ }^{2}$ \\ ${ }^{1}$ Item Plastic Surgery Clinic, Seoul; ${ }^{2}$ Department of Plastic and Reconstructive Surgery, Sanggye Paik Hospital, Inje University College of \\ Medicine, Seoul, Korea
}

Background The purpose of this study was to identify the epidemiologic characteristics of hand tendon injuries in children and to compare these with those of adults.

Methods This retrospective study was conducted on acute traumatic tendon injuries of the hand treated at our institution from 2005 to 2013, based on medical records and X-ray findings. Age, sex, hand injured, mechanism of injury, tendons and zones injured, number of affected digits, and comorbidities and complications were analyzed. Patients were divided into 2 groups: a pediatric group ( $\leq 15$ years) and an adult group ( $>15$ years).

Results Over the 9-year study period, 533 patients were surgically treated for acute traumatic tendon injuries of the hand. In the pediatric group $(n=76)$, being male, the right hand, the extensor tendon, complete rupture, the middle finger, and glass injury predominated in hand tendon injuries. In the adult group $(n=457)$, results were similar, but injury to the index finger and knife injury were the most common. An accompanying fracture was more common in the adult group and complication rates were non-significantly different.

Conclusions This comparative analysis revealed no significant epidemiologic intergroup differences. The belief that pediatric tendon injuries tend to be less severe is misplaced, and careful physical examination and exploration should be conducted in pediatric cases of hand injury.

Keywords Child / Tendons / Hand / Wounds and injuries / Epidemiologic studies
Correspondence: Young Joon Kim Department of Plastic and Reconstructive Surgery, Sanggye Paik Hospital, Inje University College of Medicine, 1342 Dongil-ro, Nowon-gu, Seoul 01757, Korea

Tel: $+82-2-950-1048$

Fax: +82-2-932-6373

E-mail:S2639@paik.ac.kr
No potential conflict of interest relevant to this article was reported.

\section{INTRODUCTION}

Various studies on hand injuries have existed for some time. In a study by the Yale-New Haven Hospital emergency service, 1,164 patients presented with hand injuries over a period of 4 months. Lacerations were the most frequent type of injury $(\mathrm{n}=716,61.5 \%)$, and $6.1 \%$ of such cases were associated with deep structural injury $(\mathrm{n}=44,6.1 \%)$ and $2.2 \%(\mathrm{n}=16)$ with tendon laceration. In addition, extensor tendon injuries were more frequent than flexor tendon injuries [1].
There have been many studies conducted on hand injuries in children in addition to research results on hand injuries for all age ranges. But there has not been comparative research on the various epidemiologic factors in hand injuries examined in comparison between children and adult patient groups. In the present study, we sought to compare acute tendon injuries of the hand in adult and pediatric patients, and to identify useful characteristics of acute tendon injuries in pediatric hands. As the hospital where this study was conducted was located in a residential area of the city, most of patients visited the hospital after 
having suffered from injuries while they were in the midst of an everyday activity, and there were relatively few cases where the injuries were inflicted by an industrial or agricultural apparatus. In addition, since the area was not a specialized, but a general residential area, and thus pediatric patients were relatively common, the environment was appropriate for a comparative study between children and adults. Through this study, an extensive analysis of the hand injuries of children and adults will be made. In turn, this study aims to discuss an approach to the hand injuries of children different from that to the injuries of adults by identifying the commonalities and differences between the 2 groups, and to prevent and reduce the frequency of hand tendon injury.

\section{METHODS}

This retrospective study on acute traumatic tendon injuries of the hand sustained from 2005 to 2013 was performed by reviewing medical records and X-ray findings. A total of $533 \mathrm{pa}-$ tients were included; all were surgically treated and underwent dynamic splint rehabilitation. In the case of adults, a modified Kessler method (2- or 4-stranded core suture) was used for the operation together with an epitenon suture, and after 2 weeks of immobilization, rehabilitation was started early. On the other hand, for children, simple interrupted sutures without epitenon sutures were used for the repair, and rehabilitation was started after 4 weeks of immobilization. Ages, sex, injured hands, mechanisms of injury, injured tendons and zones, numbers of affected fingers, and comorbidities and complications were analyzed. There are not that many cases of amputation, and because these cases are complex cases with other factors mixed in, they were excluded. In adults older than 20, injuries incurred after alcohol consumption were recorded.

Patients were divided into 2 groups; a pediatric group aged $\leq 15$ years $(\mathrm{n}=76)$ and an adult group aged $>15$ years $(\mathrm{n}=$ 457). These groups were compared with respect to gender, injured hand, injury mechanism, injured tendons, tendon zones, numbers of affected digits, and presence of combined injury of a neurovascular bundle or bone. Injury mechanisms were categorized as: knife injury (knife, scissors, or any other device using for cutting), glass injury, or crush injury. The analysis was conducted using the chi-square test in PASW ver. 18.0 (IBM Corp., Armonk, NY, USA).

\section{RESULTS}

From 2005 to 2013, a total 533 patients were surgically treated in our department for acute tendon injury of the hand. Of these
533 study subjects, 76 were assigned to the pediatric group and 457 to the adult group. Mean overall patient age was 34.4 years. There were more males than females (male $[\mathrm{M}]$ :female $[\mathrm{F}]=3.26: 1)$ and right hand $(52.9 \%)$ and extensor tendon (70.0\%) injuries predominated. In the 533 study subjects, the most common cause of tendon injury was knife injury (38.5\%) followed by glass injury (34.7\%) and crush injury. Almost all were single tendon injuries and the index finger was most affected $(25.2 \%)$ and the ring finger least affected (16.0\%). Complete tendon injury occurred in 280 patients (52.5\%) (Table 1), and zone 2 flexor tendon and zone 3 extensor tendon injuries were the most common concurrent injuries (Table 2).

In the pediatric group $(n=76)$, male patients predominated in number at 50, compared to the 26 female patients ( $\mathrm{M}: \mathrm{F}=$ 1.92:1). The mean age of pediatric patients was 9.6 years old, and the distribution of patients according to the age of pediatric

\section{Table 1. Demographic data of all tendon-injured patients}

\begin{tabular}{|lc|}
\hline Variable & Number (\%) \\
\hline Sex & 533 \\
Male & $408(76.5)$ \\
Female & $125(23.5)$ \\
Injured hand & 533 \\
Right hand & $282(52.9)$ \\
Left hand & $248(46.5)$ \\
Both & $3(0.6)$ \\
Mechanism of injury & 533 \\
Knife & $205(38.5)$ \\
Glass & $185(34.7)$ \\
Crush & $139(26.1)$ \\
Unknown & $4(0.7)$ \\
Flexor/Extensor tendon & 533 \\
Flexor tendon & $110(20.6)$ \\
Extensor tendon & $373(70.0)$ \\
Combination injury & $50(10.4)$ \\
Complete/Partial rupture & 533 \\
Complete rupture & $280(52.5)$ \\
Partial rupture & $219(41.1)$ \\
Mixed type rupture & $34(6.4)$ \\
Number of injured tendons & 533 \\
1 & $473(88.7)$ \\
2 & $39(7.3)$ \\
3 or more & $21(3.9)$ \\
Affected finger & 632 \\
Thumb & $124(19.6)$ \\
Index & $159(25.2)$ \\
Middle & $142(22.5)$ \\
Ring & $101(16)$ \\
Little & $106(16.8)$ \\
Combined injury & \\
Neurovascular injury & $106(19.9)$ \\
Fracture & $74(13.9)$ \\
Complication & $43(8.1)$ \\
Yes & \\
\hline &
\end{tabular}


patients is presented in Fig. 1. In the pediatric group, right hand injuries were slightly more common and extensor tendons were injured more often; over half of tendon injuries were complete (53.9\%) and in almost all cases, injury involved one digit (89.5\%). The most affected digit was the middle finger (25.3\%), followed by the thumb (21.9\%) and the index finger (19.8\%). Glass injury was the most common cause (38.1\%), followed by knife injury (31.6\%) and crush injury (30.3\%). Regarding accompanying injuries, 13 patients (17.1\%) had a neurovascular injury and 4 patients (5.3\%) had a fracture or bone injury (Table $3)$. In children with a flexor tendon injury, zone 2 injuries were most common (44.3\%), and in those with an extensor tendon

\section{Table 2. Specific extensor and flexor tendon zone injuries}

\begin{tabular}{|lc|}
\hline Variable & Number (\%) \\
\hline Extensor tendon zone & 486 \\
Thumb zone 1 & $26(5.3)$ \\
Thumb zone 2 & $15(3.1)$ \\
Thumb zone 3 & $24(4.9)$ \\
Thumb zone 4 & $31(6.4)$ \\
Thumb zone 5 & $12(2.5)$ \\
Zone 1 & $61(12.6)$ \\
Zone 2 & $14(2.9)$ \\
Zone 3 & $106(21.8)$ \\
Zone 4 & $42(8.6)$ \\
Zone 5 & $96(19.8)$ \\
Zone 6 & $50(10.3)$ \\
Zone 7 & $9(1.9)$ \\
Flexor tendon zone & 246 \\
Thumb zone 1 & $11(4.5)$ \\
Thumb zone 2 & $14(5.7)$ \\
Thumb zone 3 & $5(2.0)$ \\
Zone 1 & $34(13.8)$ \\
Zone 2 & $129(52.4)$ \\
Zone 3 & $39(15.9)$ \\
Zone 4 & $14(5.7)$ \\
\hline
\end{tabular}

Table 3. Demographic data of pediatric and adult patients

\begin{tabular}{|c|c|c|c|}
\hline Variable & Pediatric & Adult & P-value \\
\hline Sex & 76 & 457 & \\
\hline Male & $50(65.8)$ & $358(78.3)$ & 0.017 \\
\hline Female & $26(34.2)$ & $99(21.7)$ & \\
\hline Injured hand & 76 & 457 & \\
\hline Right hand & $42(55.3)$ & $240(52.5)$ & 0.657 \\
\hline Left hand & $34(44.7)$ & $214(46.8)$ & \\
\hline Both & 0 & $3(0.7)$ & \\
\hline Mechanism of injury & 76 & 457 & \\
\hline Knife & $24(31.6)$ & $181(39.6)$ & 0.495 \\
\hline Glass & $29(38.1)$ & $156(34.1)$ & \\
\hline Crush & $23(30.3)$ & $116(25.4)$ & \\
\hline Unknown & 0 & $4(0.9)$ & \\
\hline Flexor/Extensor tendon & 76 & 457 & \\
\hline Flexor tendon & $23(30.3)$ & $87(19.0)$ & $<0.001$ \\
\hline Extensor tendon & $38(50.0)$ & $335(73.3)$ & \\
\hline Combination injury & $15(19.7)$ & $35(7.7)$ & \\
\hline Complete/Partial rupture & 76 & 457 & \\
\hline Complete rupture & $41(53.9)$ & $239(52.3)$ & 0.790 \\
\hline Partial rupture & $31(40.8)$ & $188(41.1)$ & \\
\hline Mixed type injury & $4(5.3)$ & $30(6.6)$ & \\
\hline Number of injured tendons & 76 & 457 & \\
\hline 1 & $68(89.5)$ & 405 (88.6) & \\
\hline 2 & $3(3.9)$ & $36(7.9)$ & 0.828 \\
\hline 3 or more & $5(6.6)$ & $16(3.5)$ & \\
\hline Affected finger & 91 & 541 & \\
\hline Thumb & $20(21.9)$ & 104 (19.2) & 0.488 \\
\hline Index & $18(19.8)$ & $141(26.1)$ & \\
\hline Middle & $23(25.3)$ & $119(21.9)$ & \\
\hline Ring & $13(14.3)$ & 88 (16.3) & \\
\hline Little & $17(18.7)$ & 89 (16.5) & \\
\hline \multicolumn{4}{|l|}{ Combined injury } \\
\hline Neurovascular injury & $13(17.1)$ & $93(20.4)$ & 0.181 \\
\hline Fracture & $4(5.3)$ & 70 (15.3) & \\
\hline \multicolumn{4}{|l|}{ Complication } \\
\hline Yes & $7(9.2)$ & $36(7.9)$ & 0.693 \\
\hline
\end{tabular}

Fig. 1. Distribution of pediatric patients according to age

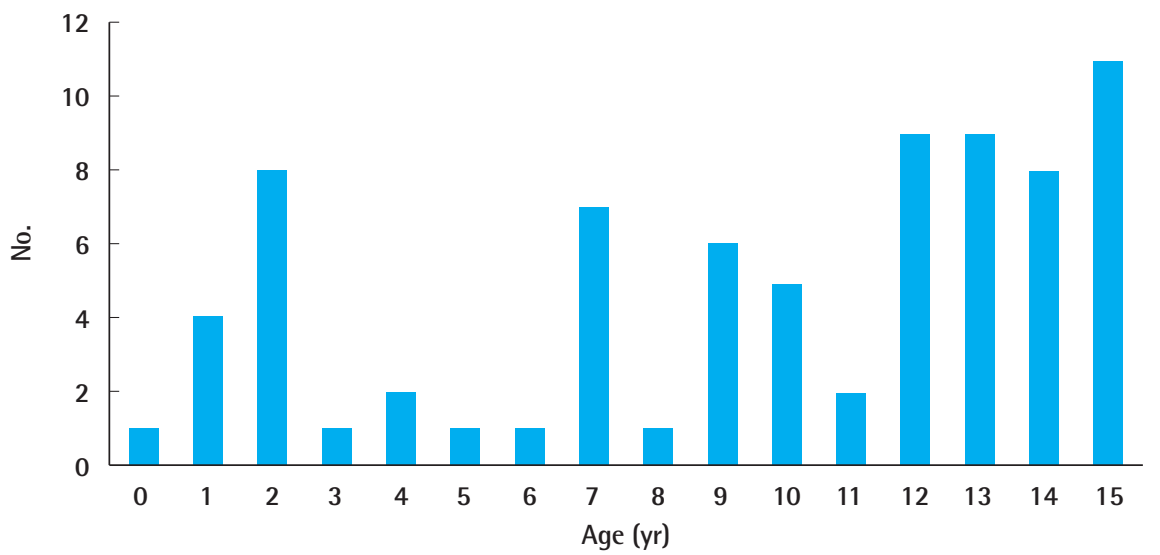


injury, zone 1 injuries occurred most often (22.2\%) (Table 4). In pediatric cases, the prevalences of knife injury were high in 2 -year-olds, in 7- to 10-year-olds, and in over 12-year-olds. The prevalence of glass injury was high in 2-year-olds and showed a rapid increase from 13 years of age. The prevalence of crush injury was relatively common in 1-year-olds and in 12- and 13-year-olds (Fig. 2).

In the adult group $(n=457)$, male patients predominated $(\mathrm{M}: \mathrm{F}=3.26: 1)$, and right hand injury, extensor tendon injury (73.3\%), and complete rupture (52.3\%) were most common.

\section{Table 4. Specific extensor and flexor tendon zone injuries} in pediatric and adult patients

\begin{tabular}{|lccc|}
\hline & Pediatric & Adult & P-value \\
\hline Extensor tendon zone & 54 & 432 & \\
Thumb zone 1 & $6(11.1)$ & $20(4.6)$ & 0.046 \\
Thumb zone 2 & $3(5.6)$ & $12(2.8)$ & 0.226 \\
Thumb zone 3 & $1(1.9)$ & $23(5.3)$ & 0.500 \\
Thumb zone 4 & $2(3.7)$ & $29(6.7)$ & 0.560 \\
Thumb zone 5 & $1(1.9)$ & $11(2.6)$ & $>0.999$ \\
Zone 1 & $12(22.2)$ & $49(11.3)$ & 0.023 \\
Zone 2 & $1(1.9)$ & $13(3.0)$ & $>0.999$ \\
Zone 3 & $6(11.1)$ & $100(23.1)$ & 0.043 \\
Zone 4 & $2(3.7)$ & $40(9.3)$ & 0.207 \\
Zone 5 & $10(18.5)$ & $86(19.9)$ & 0.809 \\
Zone 6 & $5(9.3)$ & $45(10.4)$ & 0.792 \\
Zone 7 & $5(9.3)$ & $4(1.0)$ & 0.001 \\
Flexor tendon zone & 61 & 185 & \\
Thumb zone 1 & 0 & $11(6.0)$ & 0.070 \\
Thumb zone 2 & $3(4.9)$ & $11(6.0)$ & $>0.999$ \\
Thumb zone 3 & $3(4.9)$ & $2(1.1)$ & 0.099 \\
Zone 1 & $6(9.8)$ & $28(15.1)$ & 0.298 \\
Zone 2 & $27(44.3)$ & $102(55.1)$ & 0.14 \\
Zone 3 & $9(14.8)$ & $30(16.2)$ & 0.786 \\
Zone 4 & $13(21.3)$ & $1(0.5)$ & $<0.001$ \\
\hline Values are presented as number (\%). & & \\
\hline
\end{tabular}

However, knife injury (39.6\%) was the most common cause and the index finger $(26.1 \%)$ was the most affected digit. More adult cases showed combined fracture (15.3\%) (Table 3). Extensor tendon injury occurred most commonly in zone 3 (23.1\%) and flexor tendon injury most commonly in zone 2 (55.1\%). Trauma occurred after alcohol consumption in 110 patients $(24.1 \%)$, and in these, glass injuries were most common (75.5\%). Collected data are presented in Tables 3 and 4.

Comparisons of the pediatric and adult groups revealed some differences. There was a greater proportion of female patients in the pediatric group $(\mathrm{P}=0.017)$ and flexor tendon injury and combined flexor/extensor tendon injury were more common. However, fracture $(\mathrm{P}=0.019)$ and extensor tendon injury $(\mathrm{P}<0.001)$ were less common in the pediatric group. Complication rates, including re-rupture of repaired tendon and adhesion, were non-significantly different (Table 3 ). Zone 1 injuries of the extensor tendon were more frequent in the pediatric group ( $\mathrm{P}=0.046$ for thumbs, $\mathrm{P}=0.023$ for fingers). Furthermore, injuries near the wrist were more common in the pediatric group, for extensors and flexors (extensor zone $7, \mathrm{P}=0.001$; flexor zone 4, $\mathrm{P}<0.001$ ) (Table 4).

\section{DISCUSSION}

In the present study, the higher number of male patients both among adults and children could be attributed to the fact that males were more active and exposed more frequently to environments where the injuries might be inflicted than females. The right hand had more injuries since patients appeared to be more right-handed, and the injury rate of the extensor tendon was higher since the back of the hand would be externally exposed, and thus more prone to injuries. During trauma, that tendon can be ruptured, and there are more cases of complete

\section{Fig. 2. Age and mechanism in pediatric group}

Analysis of pediatric hand injuries with respect to age and mechanism.

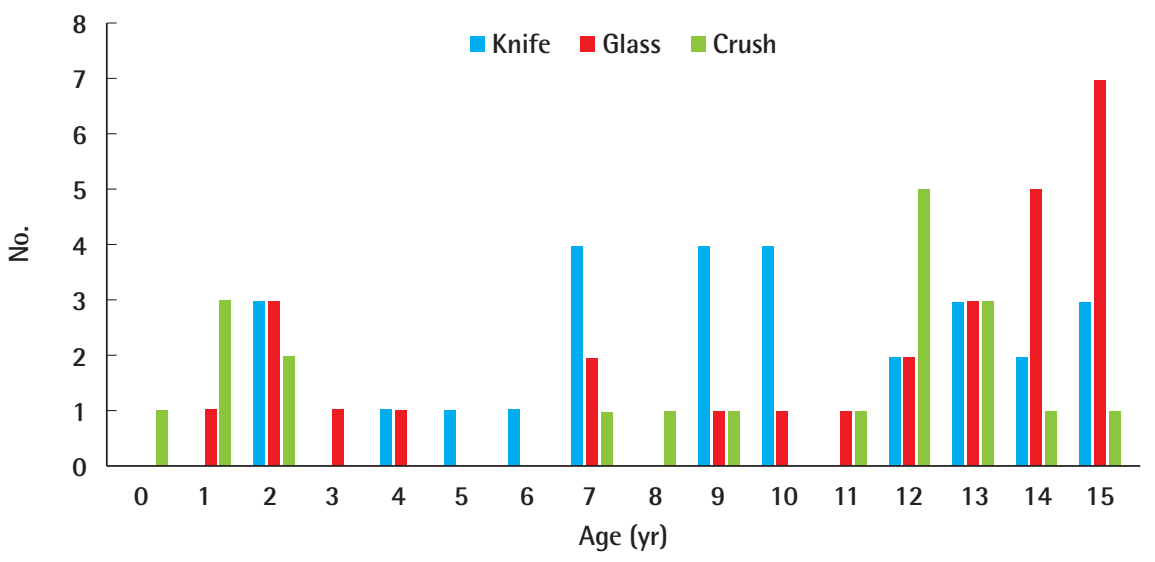


rupture than partial rupture, in which a part of the tendon still remains attached. Damaging 2 or more tendons requires a large and wide infliction of force, and the number of these cases is expected to be no greater than those in which only 1 tendon suffers a simple injury.

Since there are relatively more cases of unintended injuries due to carelessness or lack of awareness in children than those due to intentional activities, as is more common in adults, the injury rate due to glass is high, and the rate of middle finger injury is higher than that of the index finger, which is frequently used. In addition, children are involved in more cases of inadvertently grabbing hazardous materials than adults, and subsequently, they have more flexor tendon injuries (flexor tendon injury and combined flexor and extensor tendon injury).

Also, in the pediatric study, all 3 mechanisms (knife, glass, and crush injury) of hand injury occurred at high rates in 1- or 2-year-olds. The knife injury rate was also high in 7- to 10-yearolds, and rates of knife and glass injuries were an increasing trend from age 12 onward. The crush injury rate was also high in 12- and 13-year-olds. These high rates in 1- and 2-year-olds were probably due to active developmental engagement in activities and investigation of surrounding objects. Furthermore, a lack of cognitive ability in these children inevitably means that accidents are more likely. The high incidence of knife injuries observed for 7- to 10-year-olds was probably due to an increase in school activity. The higher prevalences of all 3 mechanisms of hand injury in adolescents after the middle school period (after 12 years of age) is attributed to physical violence and carefree attitudes.

In the case of adult patients, hand injuries show similar trends to those of pediatric patients, but there are a few differences. Since adults have more injuries than children from intentional activities using tools such as knives and machines, it appears that they suffer a great number of injuries by the 'knife' mechanism and more injuries on index fingers, which are used more frequently than middle fingers.

Rates of combined bone injury were significantly different, presumably because of intrinsic differences between the bones of children and adults and because children are less exposed to machines [2,3]. In addition, in children, wounds were more distally and more proximally located in the hand, and rates of injury were higher in zones 1 and 7 for extensor tendon injuries and in zone 4 for flexor tendon injuries. We are unable to offer explanations for these differences due to data inadequacies.

On the other hand, rates of accompanying neurovascular injuries were no different in the pediatric and adult groups. There is a tendency to consider wounds to be less severe in children. However, our results indicate that the severities of injuries in these 2 groups were similar. This is important, because children cooperate less during physical examinations and in many cases this makes diagnosis difficult [4]. Nevertheless, if treatment is delayed, the risk of functional disturbance is increased. This finding suggests the caution that pediatric patients with an injured hand should be examined carefully with the naked eye, or, otherwise, be diagnosed accurately using equipment such as ultrasound for possible tendon injury and combined tendon/ neurovascular injury.

For tendon repair in adults, multi-stranded sutures and epitendinous repair are preferred, since strength grows as the number of core sutures increases, and epitendinous repair results in not only a smooth repair edge but also the effect of a $50 \%$ increase in strength [5]. However, in case of pediatric patients, not only is the size of the tendon small, but also complications such as deformation of the pulley system may occur if the repaired site becomes bulkier. Therefore, Sikora et al. [6] has suggested that simple and strong core tendon repair without epitendinous repair would lead to better outcomes, which subsequently has been followed as a procedure.

Also, while early exercise is recommended for adults in rehabilitation in order to prevent complications such as adhesion and to improve function [7], the dominant opinion is that there is no need for early exercise for children since there is no functional decline in children even with up to 4 weeks of immobilization [4-6,8]. In this study, the surgery of simple and strong core tendon repair was performed on pediatric patients as well, and a dynamic splint was applied after a 4-week immobilization period.

This study was undertaken to identify the epidemiologic characteristics of hand tendon injuries in children and to compare these with those of adults. Many reports have been issued on pediatric hand tendon injuries, but most reported flexor tendon injuries or extensor tendon injuries, or only reported postoperative outcomes $[2,4,6,8-10]$. Other reports included all hand injury types, including simple laceration, fracture, neurovascular injury, and tendon injury. Generally, extensor injury, right hand injury, index finger injury, and male gender are the most common factors [11]. Our results largely concur, although some differences were found. First, the prevalence of acute tendon injuries in pediatric patients was a little higher in the present study. de Jong et al. [11] reported a rate of pediatric acute tendon injuries of $5.2 \%$, whereas in the present study, the rate was higher at $14.3 \%$. Second, the rate of complete tendon rupture was much lower in the present study. Sikora et al. [6] reported in their 10year study of pediatric flexor tendon injuries the finding of a complete injury rate of $95 \%$, but in the present study, this was only $53.9 \%$. Since de Jong et al. included wrist injuries in the 
hand injuries category, it was suggested that the rate of pediatric acute tendon injuries was relatively low, while Sikora et al. analyzed only the cases where the flexor tendon was damaged, leading to the high rate of complete rupture. On the other hand, in this study, all the hand injuries in which the tendon was damaged, including the extensor tendon, were analyzed, and subsequently the rate of complete rupture was relatively low. We believe that both studies have a percentage difference from this study due to the difference in the target range of analysis, and because this study took into consideration solely all the factors of the hand that could be examined, this study is more clinically useful.

The main limitation of the present study is that the data used were obtained at one hospital, which inevitably introduces bias. Furthermore, the number of pediatric patients was too small to support subgroup analysis. Nonetheless, this study is the first to describe pediatric hand tendon injuries from an epidemiologic perspective and to provide a comparison of these injuries and those of adults. In addition, we analyzed all flexor and extensor tendon injuries at the same time and analyzed pediatric patient data with respect to age and injury mechanisms. We hope our results will aid the examination and treatment of pediatric patients with a hand tendon injury.

In conclusion, we found no evidence to suggest that pediatric hand tendon injuries are less severe than those of adults, meaning that tendon injuries in pediatric hands are as severe as those in adult hands. This finding contradicts the notion that pediatric injuries tend to be less severe than injuries in adults, and suggests that pediatric hand injuries warrant the same level of careful examination. Our findings also indicate that elementary students be given proper instructions and warned regarding the use of knives, blades, or scissors, and that adolescents should avoid dangerous materials. To prevent hand injuries in 1- to 2-yearolds, parents should be more cautious about the accessibility of glasses and knives, and more aware of crush injury hazards.

\section{REFERENCES}

1. Frazier WH, Miller M, Fox RS, et al. Hand injuries: incidence and epidemiology in an emergency service. JACEP 1978;7:265-8.

2. Valencia J, Leyva F, Gomez-Bajo GJ. Pediatric hand trauma. Clin Orthop Relat Res 2005; (432):77-86.

3. Strickland JW. Bone, nerve, and tendon injuries of the hand in children. Pediatr Clin North Am 1975;22:451-63.

4. Nietosvaara Y, Lindfors NC, Palmu S, et al. Flexor tendon injuries in pediatric patients. J Hand Surg Am 2007;32: 1549-57.

5. Lehfeldt M, Ray E, Sherman R. MOC-PS(SM) CME article: treatment of flexor tendon laceration. Plast Reconstr Surg 2008;121:1-12.

6. Sikora S, Lai M, Arneja JS. Pediatric flexor tendon injuries: a 10-year outcome analysis. Can J Plast Surg 2013;21:181-5.

7. Chesney A, Chauhan A, Kattan A, et al. Systematic review of flexor tendon rehabilitation protocols in zone II of the hand. Plast Reconstr Surg 2011;127:1583-92.

8. Berndtsson L, Ejeskar A. Zone II flexor tendon repair in children: a retrospective long term study. Scand J Plast Reconstr Surg Hand Surg 1995;29:59-64.

9. Grobbelaar AO, Hudson DA. Flexor tendon injuries in children.J Hand Surg Br 1994;19:696-8.

10. Kato H, Minami A, Suenaga N, et al. Long-term results after primary repairs of zone 2 flexor tendon lacerations in children younger than age 6 years. J Pediatr Orthop 2002;22: $732-5$.

11. de Jong JP, Nguyen JT, Sonnema AJ, et al. The incidence of acute traumatic tendon injuries in the hand and wrist: a 10year population-based study. Clin Orthop Surg 2014;6:196202. 